\section{Commentary: The surgeon and the tailor. A continuous partnership}

\author{
Ruggero De Paulis, MD
}

The proper treatment of acute type A aortic dissection remains a surgical challenge that presents significant operative mortality. In this peculiar emergency clinical condition, both surgical dexterity and surgical strategy play an important role in the final result. Different clinical presentations associated with variable anatomical tissue destruction explain the significant difference in mortality and overall results that are present in the majority of the reports in the literature.

Lau and colleagues ${ }^{1}$ report their personal experience in a substantial number of patients over a 22 -year period. In two-thirds of cases, they adopted a conservative strategy of root sparing and hemiarch, whereas in the remaining cases a more extensive root and/or arch replacement was carried out. A careful tailoring of the operation to a patient's baseline profile in term of age and associated comorbidities, coupled with clinical and anatomical features at presentation, was instrumental in achieving an extremely low operative mortality and outstanding overall results, irrespective of the conservative or extensive form of treatment.

The importance of a conceptual approach is especially important in this specific type of pathology where the correct evaluation of the risk to benefit ratio needs to be immediately correlated with the extent of the surgery that is awaiting the patient. A perfect but extensive and long surgery might not be endured by an old and frail patient; alternatively, a conservative surgery might not be resolutive for a young patient with connective tissue disease. The higher incidence of major adverse events and major adverse pulmonary events despite a conservative approach, further highlight the right choice of limiting the influence of

From the Deptartment of Cardiac Surgery, European Hospital, Unicamillus International Medical University, Rome, Italy.

Disclosures: The author reported no conflicts of interest.

The Journal policy requires editors and reviewers to disclose conflicts of interest and to decline handling or reviewing manuscripts for which they may have a conflict of interest. The editors and reviewers of this article have no conflicts of interest.

Received for publication Dec 20, 2020; revisions received Dec 20, 2020; accepted for publication Dec 21, 2020; available ahead of print Dec 25, 2020.

Address for reprints: Ruggero De Paulis, MD, Department of Cardiac Surgery, European Hospital, Via Portuense 700, 00149 Rome, Italy (E-mail: rdepaulis58@gmail. com).

J Thorac Cardiovasc Surg 2022;164:1708-9

$0022-5223 / \$ 36.00$

Copyright (c) 2020 by The American Association for Thoracic Surgery

https://doi.org/10.1016/j.jtcvs.2020.12.087

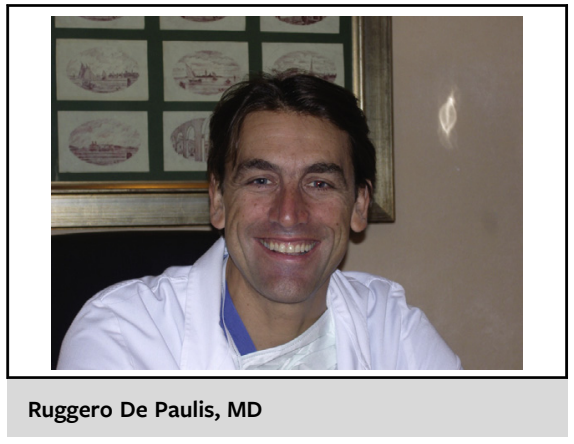

CENTRAL MESSAGE

Tailoring the right operation for

the right patients contributes to

better results. However, specific

anatomical presentation might

prevent choice by requiring a

determined surgical approach.

surgery in a population at higher risk. However, a proper surgical strategy is only 1 face of the coin. Once the correct strategy has been put into action, perfect execution of the operation is of paramount importance. It is well known that a conservative approach might fail for recurrent root dissection with valve prolapse and consequent insufficiency, or for progressive arch enlargement due to the continuous perfusion of the false channel through the pinhole entry tears of the distal suture line. A conservative operation, apparently simple, still requires the necessary experience to guarantee stable and lasting results. To this extent, Lau and colleagues ${ }^{1}$ should be congratulated for achieving good 10-year results in their conservative approach without the help of glue or the use of Teflon (Chemours, Newark, Del) felt within the dissected layers.

Another example of good tailoring, just like the higher incidence of major adverse events and major adverse pulmonary events in patients with conservative approach, is the greater rate of late distal reintervention in patients with extensive surgery at the index operation. In this context it represents a marker for a more severe pathology rather than an incomplete treatment.

Tailoring is a good strategy but requires fine clinical judgment, uniform standards in decision making, a good amount of experience, and a consistent surgical technique. Nevertheless, the anatomical presentation often takes the lead 
and indicates the road. What if a frail, old patient presents with a dilated and dissected root with a tear into an already aneurysmatic arch? The tailor watches the surgeon who takes his or her time and goes out in a more difficult situation of a root and arch replacement.
See Article page 1698.

\section{Commentary: The human factor in repair of acute dissection}

\author{
Amedeo Anselmi, MD, PhD \\ "A perfection of means, and confusion of aims, seems \\ to be our main problem."
}

\section{—Albert Einstein}

Advances in our discipline have occurred at an increasing pace during the past decade: technologies enabling reliable transcatheter and hybrid valve therapies, numbers of novel devices (mainly for transcatheter/hybrid applications) being conceived, improvement/standardization of existing surgical techniques/prostheses, diffusion of minimally invasive approaches, artificial intelligence, computer-assisted surgery, and so on. Many innovations shall not attain the stage of routine clinical application, yet major investments continue to be poured into the most promising fields. Surgery of the thoracic aorta has participated in this evolution; for example, with different branched or hybrid prostheses adapting to variable anatomies for extensive aortic replacement in 1 operation, refined brain and organ protection (selective antegrade cerebral perfusion representing a powerful and nearly unmissable tool), multiple options for

\footnotetext{
From the Division of Thoracic and Cardiovascular Surgery, Pontchaillou University Hospital, Rennes, France.

Disclosures: The author reported no conflicts of interest.

The Journal policy requires editors and reviewers to disclose conflicts of interest and to decline handling or reviewing manuscripts for which they may have a conflict of interest. The editors and reviewers of this article have no conflicts of interest.

Received for publication Jan 17, 2021; revisions received Jan 17, 2021; accepted for publication Jan 20, 2021; available ahead of print Jan 28, 2021.

Address for reprints: Amedeo Anselmi, MD, PhD, Division of Thoracic and Cardiovascular Surgery, Pontchaillou University Hospital, 2 Rue Henri le Guilloux, Rennes, France (E-mail: amedeo.anselmi@chu-rennes.fr).

J Thorac Cardiovasc Surg 2022;164:1709-10

$0022-5223 / \$ 36.00$

Copyright (C) 2021 by The American Association for Thoracic Surgery

https://doi.org/10.1016/j.jtcvs.2021.01.071
}

\section{Reference}

1. Lau C, Robinson B, Farrington WJ, Rahouma M, Gambardella I, Gaudino M, et al A tailored strategy for repair of acute type A aortic dissection. J Thorac Cardiovasc Surg. 2022;164:1698-707.e3.

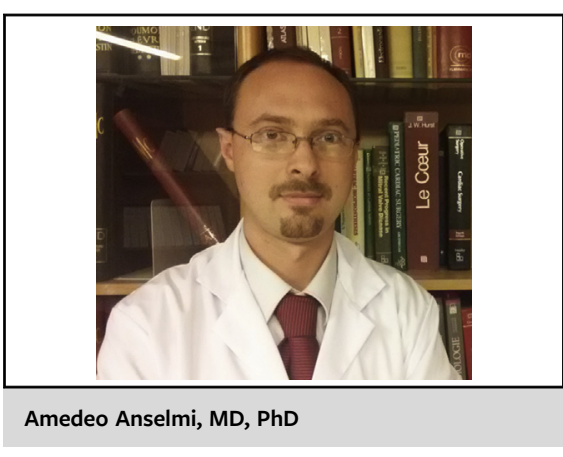

CENTRAL MESSAGE

Reasonable clinical judgment is the compass for operative management of type A dissection. Nonetheless, this proficiency requires substantial experience and its transmission is not easily obtained.

root management, and endovascular and hybrid repair. This has occurred to the great benefit of many patients.

In this context, decision making about the most adapted management strategy in individual patients, and the relative role of alternative techniques, represents the main feature and challenge of current cardiac surgery practice, to the point of redefining the role of cardiac surgeons within Heart Teams. The current, remarkable article from Lau and colleagues at Weill Cornell University ${ }^{1}$ reminds us that our philosophical approach should be patient-oriented rather than disease-oriented, and that the goal of surgery for acute type A dissection is to save a patient's life in an emergency setting. The risks of the operation should undoubtedly be balanced against the patient's life expectancy and overall profile. The performance of an extensive repair at primary surgery (at the aortic root and/or arch) represents a marker of greater aggressiveness of the disease. As such, it is associated with an increased need for reintervention at followup. Conservative repair patients were older in the current 